\title{
Real-Time HALCON-based Pose Measurement System for An Astronaut Assistant Robot
}

\author{
Lihong Dai ${ }^{1,2,3}$, Jinguo Liu ${ }^{1 *}$, Zhaojie $\mathrm{Ju}^{4,1}$,Yuwang Liu ${ }^{1}$ \\ ${ }^{1}$ State Key Laboratory of Robotics, Shenyang Institute of Automation, Chinese Academy of \\ Sciences, Shenyang 110016, China \\ E-mail: dailihong2004@163.com \\ * Corresponding author, Phone: 024-23970712, E-mail: liujinguo@ sia.cn \\ E-mail: liuyuwang@sia.cn \\ ${ }^{2}$ School of Electronic and Information Engineering, University of Science and Technology \\ Liaoning, Anshan 114051, China \\ ${ }^{3}$ University of the Chinese Academy of Sciences, Beijing 100049, China \\ ${ }^{4}$ Intelligent Systems \& Biomedical Robotics, School of Creative Technologies University of \\ Portsmouth, UK \\ E-mail:zhaojie.ju@port.ac.uk
}

\begin{abstract}
The manned space program in China has entered the space station stage. Space astronauts normally have very limited time for certain tasks, so in order to free space astronauts from some repetitive routine tasks, various robots in space appear. Free-flying robots in space can also provide conditions for various scientific experiments with their unique microgravity advantages. The measurement of their positions and attitudes is the premise of their autonomous flight and remote operations. So, the pose measurements system of the Astronaut Assistant Robot is designed in this paper. With the camera calibrated, the image is acquired and processed in real time, and the robot is tracked in real time. In the end poses are estimated by virtue of $\mathrm{PnP}$ algorithm. The pose measurements system is modeled and simulated, with an automatic global threshold method to improve the reliability of the system. In addition, a series of measures are adopted to improve its efficiency with a satisfactory accuracy. The experimental results show that the measurement system meets the requirements of both effectiveness and efficiency.
\end{abstract}

Keywords: Astronaut Assistant Robot, Pose Measurement System, PnP Algorithm, Real Time

\section{$1 \quad$ Introduction}

China's manned space program has entered the space station phase ${ }^{[1]}$. Compared with the ground laboratory, the space station can provide unique microgravity environment for science research to obtain the results which are difficult to get from the ground station. Astronauts in the space shuttle normally have limited time for certain tasks. Robots in space can help astronauts to do some repetitive work and provide support 
for astronauts. And free-flying robots in space normally do not touch the capsule, so there is no mechanical vibration, which can provide a kind of microgravity environment. On the one hand, only small power is needed to control their operation. On the other hand, microgravity environment needed for the scientific experiment is provided. It can be seen that the free-flying robots in space play an important role in the space cabin. There are various free-flying in-cabin robots, such as SPHERE ${ }^{[2]}$ developed by the university of MIT; PSA, Smart SPHERE ${ }^{[3]}$ and Astrobee ${ }^{[4-5]}$ developed by the NASA AMS research center; SHB developed in Japan; SCAMP developed by the university of Maryland in the United States; Mini AERCam developed at NASA Johnson space center; and AAR ${ }^{[6]}$ (The first generation in-cabin robot) and AAR-2 ${ }^{[7]}$ (The second generation in-cabin robot) developed by Shenyang institute of automation, Chinese academy of sciences.

SPHERE ${ }^{2]}$ is mainly used for formation flight and docking, where dynamic programming method is adopted. However, its beacon-based localization limit the operating space. Based on SPHERE, Smart SPHERE is installed a commercial smartphone as an embedded controller to improve performance, which can capture images using embedded camera in smartphone. Astrobee ${ }^{[4-5]}$ is developed recently. Compared with SPHERE, Astrobee extends the space largely, by using the tracking and positioning method of monocular vision. But in measurement system, SURF feature detection and BRISK feature matching algorithms are used, which are more complex, limiting its efficiency. AAR ${ }^{[6]}$ is controlled by PID Network algorithm, and is positioned by binocular visual system. Based on AAR of the first generation incabin robot, AAR-2 ${ }^{[7]}$ is designed and developed, which is controlled by using the fuzzy sliding mode control algorithm. There are few detailed descriptions of the pose measurement system on robots in space in literatures. However the measurement of their position and attitude is the premise of their autonomous flight and remote operation. So, the pose measurement system on AAR-2 is designed in the paper. The picture of AAR-2 is shown in Fig. 1.

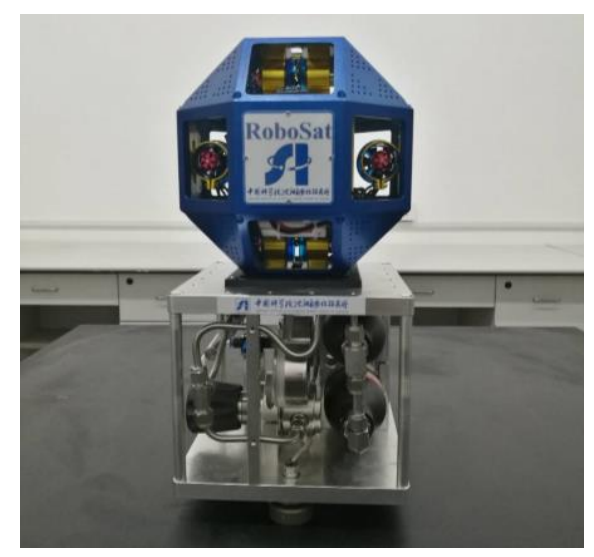

Fig.1 The picture of AAR-2 
Monocular vision measurement system has the advantages of simple structure, convenient operation, easy calibration, and fast calculation speed. So it is suitable for the real-time application. In this paper, the method based on monocular vision is used in position and attitude measurement system.

\section{Pose Measurement System}

A camera is mounted on an AAR-2, so it moves with AAR-2. The target is installed in the inner wall of the cabinet or sealed cabin, with known feature point distribution patterns. The image of target is acquired in real-time by the camera. A series of measurement is taken in order to improve the performance of real-time. Furthermore, the system is simulated by the powerful HALCON visual software.

The pose measurement system mainly can be divided into five sections: camera calibration, image acquisition, image processing, feature extraction and pose calculation, as shown in Fig.2.

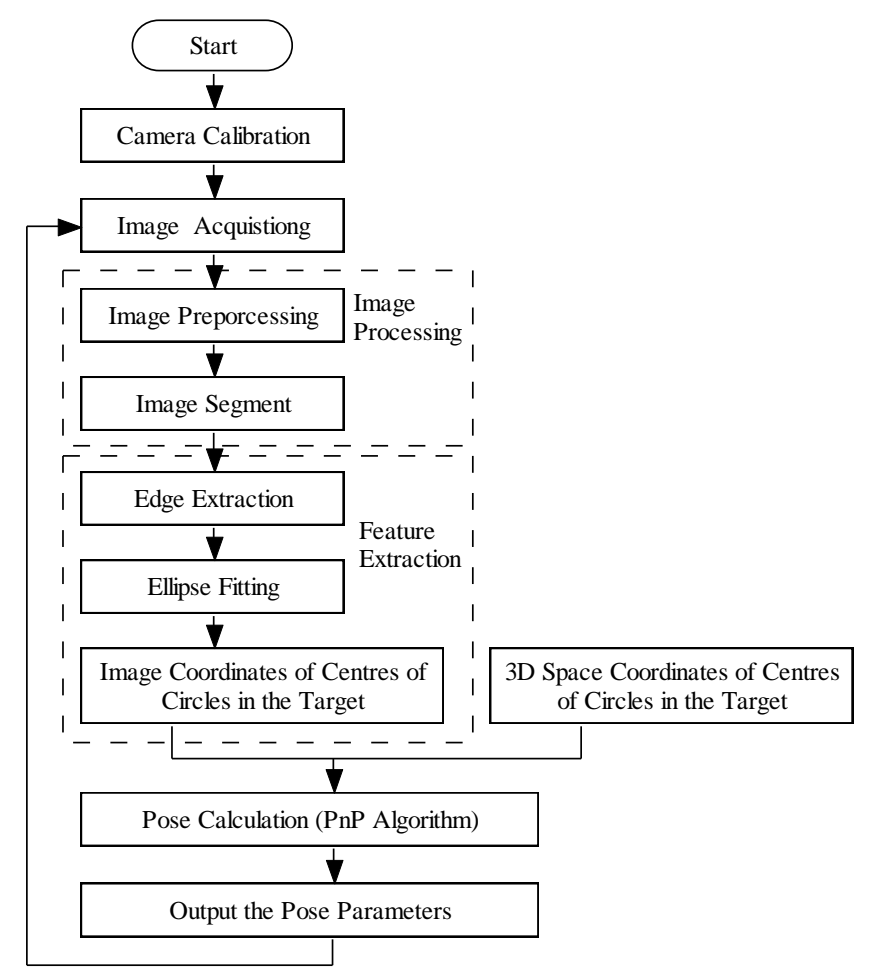

Fig.2 Schematic diagram of the pose measurement system

The purpose of the camera calibration is to calculate the interior camera parameters which provide the basic data for pose calculation of camera. After image acquisition, image processing and feature extraction are carried out. Image processing mainly 
includes image preprocessing and image segmentation. And feature extraction mainly includes edge extraction, elliptical fitting and coordinate extraction of target center. On the basis of 2D image coordinates of target circles centers and their 3D space coordinates, the positon and attitude parameters of the camera relative to cooperative target are calculate with $\mathrm{PnP}$ algorithm, which are saved to a file, and provide the foundation for better control and operation of the AAR-2.

\subsection{Camera Calibration}

In order to calculate the position and attitude more accurately, it is necessary to calibrate the camera first. The camera calibration model is set up, the method of camera calibration proposed by Zhengyou Zhang is employed, and the process of the camera calibration is realized by virtue of the HALCON software.

Firstly, when the distortion of camera lens is not considered, the camera model is the ideal small pinhole imaging model. The conversion process of 3D world coordinates to 2D image pixel coordinates is obtained through the camera model, in which the parameters of the camera is involved. The camera calibration process is that of determining the camera parameters.

Then, in the plane template calibration method proposed by Zhengyou Zhang ${ }^{[8]}$, the world coordinate system is set on the plane of the calibration plate. The interior camera parameters and the distortion coefficient obtained are computed as the initial values. And the parameters with high final accuracy are obtained by the method of levenberg-marquarat nonlinear optimization.

HALCON has been recognized as the most effective machine vision software in industry in Europe and Japan. It provides some assistants and visual tools, as well as programming hints, which make programming and modification easy, development cycles short, and development costs low. It is widely used to develop visual system, such as in the literatures ${ }^{[9-10]}$. The process of calibrating camera is as follows. Calibration data model is created at first. Initial camera parameters are set in the calibration data model. Then calibration object is set. The image of calibration plate is read to find the coordinate of marks and pose of target, which are saved in data model. Next, camera is calibrated to get the interior camera parameters. Finally the interior camera parameters is written in files.

\subsection{Image Acquisition}

The image acquisition assistant in HALCON can be used for real-time image acquisition. If you connect the camera to the computer via the USB port, and then click "the image acquisition assistant" in the assistant menu, an image acquisition window will pop up. Under the resource tab, the camera can be detected by clicking on "the image acquisition interface" radio button. And under the connection tab, the connected camera device can be seen. Furthermore, under the code generation tab, if you click "insert code" button, image acquisition code can be generated automatically, which makes programming easy. 


\subsection{Image Processing}

(1) Image Preprocessing.

In order to improve the anti-interference ability of the image, and decrease the noise in the image, image often is preprocessed with filter.

Filtering is classified into time domain filtering and frequency domain filtering. Image filtering in time domain includes linear filtering and nonlinear filtering. The common linear filtering mainly includes mean filtering and Gaussian filtering. The nonlinear filtering mainly includes median filtering, bilateral filtering and anisotropic diffusion filtering. Because the frequency domain filtering is processed by the Fourier transform and inverse transform, and the nonlinear filtering is often complicated, which are not conducive to the real-time processing of the image, the linear filter is selected in the paper. In the linear filter, the mean filter is the average gray value of the pixel in the template, whose filtering effect is often not very ideal. The Gaussian filter is a Gaussian weighted average gray value of pixel in the template. Most of image signal or energy concentrated in low frequency and medium frequency amplitude spectrum, while at higher frequencies, useful information is often submerged by noise, and so one filter which can reduce high frequency amplitude can reduce noise. Gaussian filtering is essentially a low-pass filter, which is widely used in image denoising and is very effective in suppressing the noise that obeys normal distribution. The image was pretreated with function named gauss_image in HALCON.

(2) Image Segmentation

The gray threshold segmentation method is most applied in image segmentation. It is a method to separate the object from the background by threshold value, whose advantages are simple calculation, high efficiency and fast speed. It is widely used in real-time.

It can be seen that the key of threshold segmentation algorithm is to determine the threshold, so if a suitable threshold is determined, the image can be separated accurately. Because the actual target image shown in Fig. 3 has a single background, and the difference between foreground and background is obvious which can be seen from the gray histogram shown in Fig. 4, there is no need for complex threshold segmentation method, and the global threshold can be set for image segmentation.

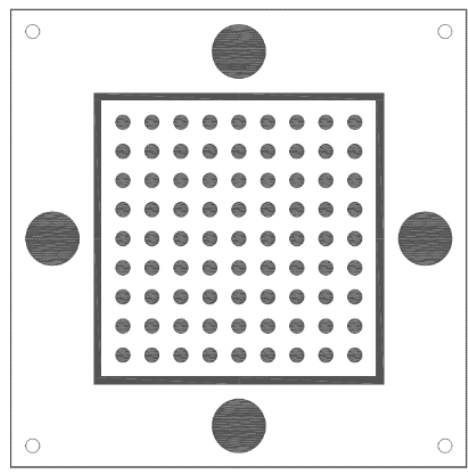

Fig. 3 Image of cooperative target 


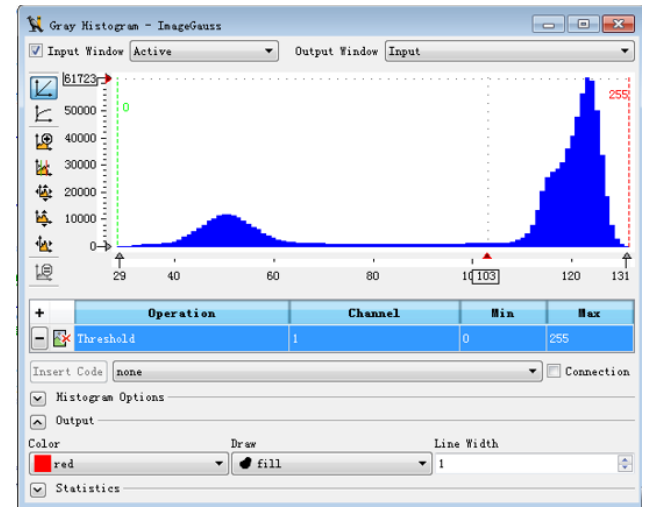

Fig. 4 Gray histogram

In order to improve reliability, the automatic global threshold method is adopted to image segmentation. Firstly, the histogram of image is counted. Then, the highest frequency of gray values is found. Finally, the grayscale value lower than a certain amount of gray value at the highest frequency is considered as the threshold. The corresponding instruction in HALCON is as follows.

gray_histo(ImageGauss,ImageGauss,AbsoluteHisto,RelativeHiso)

PeakGray:=sort_index(AbsoluteHisto)[255]

threshold(ImageGauss, Regions,0,PeakGray*0.85)

After selecting the foreground from the background in the target image, the marker circle region can be chosen mainly according to difference between the area of the marker circles and other objects, which can be realized by using the visual interface of the feature histogram in HALCON. Then, using the cutout technique ${ }^{[11]}$, we extracted the marked circle region in the original image, so that the marked circle image was extracted.

(3) Morphological Image Processing.

The methods constantly used in morphological image processing are dilation operation, corrosion operation, open operation and closed operation. The dilation operation makes object larger and is used to fill the holes and narrow gaps in the objects. In contrast, Corrosion can be used to eliminate small and meaningless objects. Open operation is dilation after corrosion, and its effect is similar to that of corrosion, which reduces the object. It has less effect on object reduction than corrosion operation. Closed operation is corrosion after dilation, and its effect is similar to that of dilation operation, which makes the object bigger. It has less effect on object expansion than dilation operation.

In order to better extract the edge of the circle in the target image, the closed operation is used to increase the circle area appropriately. The function named "closing circle" in HALCON is used. Because the extracted objects are circles, the structure element is chosen as circle. 


\subsection{Feature Extraction}

After image preprocessing and image segmentation, the image of marker circle is determined. Next, edge detection is needed to obtain the edge of marker circles. And then they are fitted into ellipse in order to determine the coordinates of the circles center.

(1) Image Edge Detection

Edge of image is set of pixels whose grey value take on step change, so we can detect the image edge by calculating the maximum value of first-order derivative. Gradient operator commonly used based on the first derivative includes Sobel, Roberts, Prewitt. In addition, image edge can be detected by zero crossing of second derivative. Operator frequently used based on the second derivative is Laplacian of a Gaussian. These operators are on the basis of local window, so their algorithms are simple and easy to implement. However, if these algorithm are adopted, some information on the edge will be lost, the system is sensitive to noise, and edge detection effect is not very ideal. In 1986 John Canny proposed a new edge detection operator $^{[12]}$. The operator is with the optimization idea, which has a large signal-to-noise ratio and a higher detection precision. It is currently considered as the most ideal edge detection method, and widely used ${ }^{[13-14]}$. The process of Canny edge detection is as follows.

(1) The image is smoothed with a Gaussian filter to remove noise and interference. One-dimensional Gaussian function is used as filter. The original image $f(x, y)$ is convolved by row and column respectively. The image after smoothing is $I(x, y)$. The Gaussian filter and $I(x, y)$ are given by

$$
\begin{gathered}
G(x)=\frac{\exp \left(-x^{2} / 2 \sigma^{2}\right)}{2 \pi \sigma^{2}} \\
I(x, y)=[G(x) G(y)]^{*} f(x, y)
\end{gathered}
$$

where $\sigma$ is the standard deviation of Gaussian, which is used to control the smoothness.

(2) For the smoothed image $I(x, y)$, the finite difference of first-order partial derivatives is calculated, and the amplitude $\mathrm{M}$ and the direction $\theta$ of the gradient are obtained, given by

$$
\begin{gathered}
\left\{\begin{array}{c}
P_{x}[i, j]=(I[i+1, j]-I[i, j]+I[i+1, j+1]-I[i, j+1]) / 2 \\
P_{y}[i, j]=(I[i, j+1]-I[i, j]+I[i+1, j+1]-I[i+1, j]) / 2
\end{array}\right. \\
M[i, j]=\sqrt{P_{x}[i, j]^{2}+P_{y}[i, j]^{2}} \\
\theta[i, j]=\arctan \left(\frac{P_{y}[i, j]}{P_{x}[i, j]}\right)
\end{gathered}
$$

(3) Non-maximum suppression is carried out. The image is scanned along the image gradient direction, and if pixels are not part of the local maxima they are set to zero. 
(4)Double threshold algorithm is used to detect and connect edges. High and low thresholds are set. The point where the gradient is greater than the high threshold is considered to be the real edge of the image, so it is retained. One where the gradient value is less than the low threshold is not edge and is removed. For the point between the two thresholds, those adjacent to the edge point are retained as edge, otherwise deleted.

Because Canny edge detection is better than other methods, it is used to detect the edge of the target circle. The instruction in HALCON is edges_sub_pix (ImageReduced, Edges, 'Canny', 4, 20, 40).

(2) Elliptic Fitting

Edge detection is used to extract edges, which reduces the amount of data, eliminates irrelevant information, and retains the important structural attributes of the image. Because the result of edge detection is a collection of edge pixels, it is also necessary to select the contour as a whole. The function used in HALCON for contour selection is select_contours_xld.

In order to calculate the centers of the target circles, their contours need to be fitted with an ellipse. Image after elliptic fitting is shown in Fig.5. Compared with the edge detected, the data is reduced. The function of the elliptic fitting contour in HALCON is fit_ellipse_contour_xld.

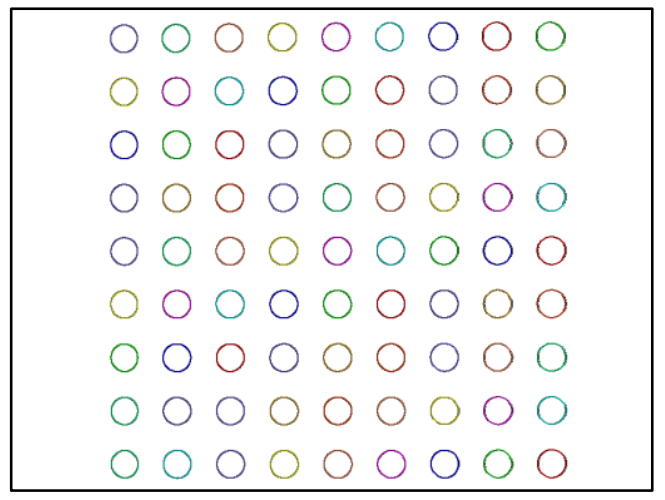

Fig. 5 Image after elliptic fitting

\subsection{Pose Calculation}

Based on the mathematical model of the camera and the known camera interior parameters, pose calculation is to establish the relationship between $3 \mathrm{D}$ space coordinates of target features and 2D coordinates of image features, so as to determine the relative position and attitude between camera and target.

The commonly used features include point features, linear features, etc. Among them, the problem of pose calculation using point feature is called PnP (perspective-npoint) problem. When $n$ of point number is less than or equal to 2, known condition is insufficient, so the pose parameters of the target cannot be determined. When $n>5$, the problem can be solved linearly. When $3 \leq \mathrm{n} \leq 5$, the PnP problem is usually nonlinear, 
and there are possible multiple solutions. Due to the requirement of real time in practical application, points between 3 and 5 are often selected to calculate the position and attitude.

The P3P problem can be described as follows. The known conditions are that angles between two of three rays starting from a vertex $O$ are $\alpha, \beta, \gamma$, and three sides of a triangle are $c, b, a$, respectively. The distance between the vertex $O$ of rays and three vertex of the triangle ABC, namely d1, d2, and $\mathrm{d} 3$, will be calculated. Description of P3P problem is shown as Fig. 6.

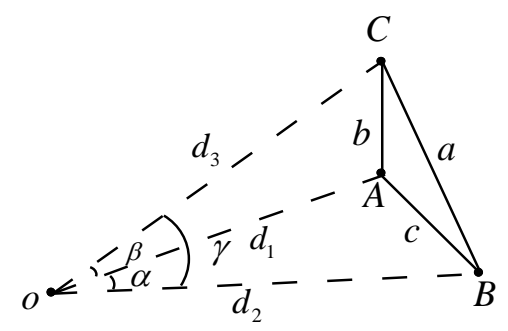

Fig. 6 Description of P3P problem

By using cosine theorem, we have

Suppose

$$
\left\{\begin{array}{l}
d_{1}^{2}+d_{2}^{2}-2 d_{1} d_{2} \cos \alpha=c^{2} \\
d_{1}^{2}+d_{3}^{2}-2 d_{1} d_{3} \cos \beta=b^{2} \\
d_{2}^{2}+d_{3}^{2}-2 d_{2} d_{3} \cos \gamma=a^{2}
\end{array}\right.
$$

$$
\left\{\begin{array}{l}
d_{2}=x d_{1} \\
d_{3}=y d_{1}
\end{array}\right.
$$

Substitute (7) into (6), we can derive

$$
\left\{\begin{array}{l}
d_{1}^{2}\left(1+x^{2}-2 x \cos \alpha\right)=c^{2} \\
d_{1}^{2}\left(1+y^{2}-2 y \cos \beta\right)=b^{2} \\
d_{1}^{2}\left(x^{2}+y^{2}-2 x y \cos \gamma\right)=a^{2}
\end{array}\right.
$$

If two of the ones in (8) are taken, and $\mathrm{d} 1$ is eliminated, we can obtain

$$
\left\{\begin{array}{c}
a^{2}\left(1+y^{2}-2 y \cos \beta\right)=b^{2}\left(x^{2}+y^{2}-2 x y \cos \gamma\right) \\
b^{2}\left(1+x^{2}-2 x \cos \alpha\right)=c^{2}\left(1+y^{2}-2 y \cos \beta\right)
\end{array}\right.
$$

Connecting two equations of (9) and removing $b^{2} x^{2}$, it is not difficult to derive that

$$
\begin{aligned}
& a^{2}\left(1+y^{2}-2 y \cos \beta\right)-b^{2}\left(y^{2}-2 x y \cos \gamma\right) \\
& =c^{2}\left(1+y^{2}-2 y \cos \beta\right)-b^{2}(1-2 x \cos \alpha)
\end{aligned}
$$

Extracting $x$ from (10), we can obtain 


$$
x=\frac{a^{2}+b^{2}-c^{2}+\left(a^{2}-b^{2}-c^{2}\right) y^{2}-2 y\left(a^{2}-c^{2}\right) \cos \beta}{2 b^{2}(\cos \alpha-y \cos \gamma)}
$$

Substituting (11) into the lower equation of (9), a quartic equation about y is obtained as

$$
a_{4} y^{4}+a_{3} y^{3}+a_{2} y^{2}+a_{1} y+a_{0}=0
$$

Four sets of solutions for $\mathrm{y}$ can be found. Substituting the solution of $\mathrm{y}$ into (11), the value of $x$ can be obtained. According the above equation of (8), we have

$$
d_{1}=\sqrt{c^{2} /\left(1+x^{2}-2 x \cos \alpha\right)}
$$

Thus $d_{1}$ can be calculated. According to (5), $d_{2}$ and $d_{3}$ can also be obtained. From the process derived above, it can be seen there are at most four solutions to the P3P problem. For P4P problem, when four control points are coplanar, there is only one solution to the problem. Any three points can be selected to determine the pose, and then the fourth point is used to verify it ${ }^{[15]}$. Since the actual target image is coplanar, four control points are used to determine the pose. The instruction of pose calculation in HALCON is vector_to_pose.

\section{Performance Evaluation}

AAR-2 above air bearing table, the MV-500SM CMOS camera, and the M1620MPW2 optical lens produced by Computar company are used in the system to carry out the simulation experiment. The resolution is $2592 * 1944$ pixels. Its pixel size is 2.2 $\mu \mathrm{m}$. The AAR-2 experimental platform is shown in Fig. 7.

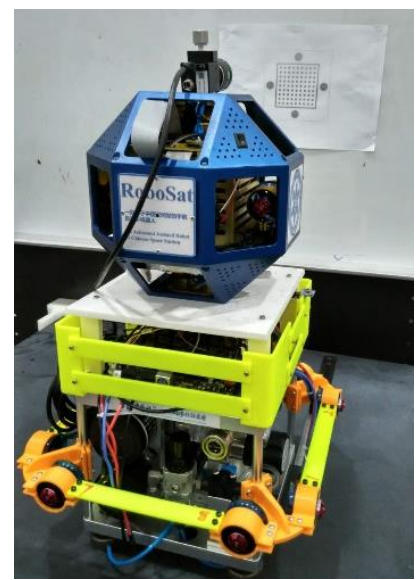

Fig.7 AAR-2 experimental platform

In order to meet the real-time demand of the system, the operation efficiency of the system can be improved by simplifying the algorithm, reducing the complexity of image processing and transformation, and reducing the storage space. There are two 
main methods: one is to reduce the amount of data to be processed; the second is to adopt a simple or simplified algorithm. Reducing the amount of data to be processed plays a major role in real-time of image processing algorithms.

The measures to reduce the amount of data to be processed are taken to improve the operation efficiency of the program in the paper.

(1)The function of image zooming is applied to reduce the size of the image from $2592 * 1944$ to $1024 * 768$, which lifts the speed of the program. The corresponding instruction in HALCON is zoom_image_size (Image 1, Image, 1024, 768, 'constant').

(2) By setting the interest area or selective region, the data volume of image processing is greatly reduced, and the operation efficiency is dramatically improved. The corresponding instruction in HALCON is reduce_domain (Image, ROI_Rectangle, ImageReduced1), where ROI_Rectangle is the area of interest, which include 9 circles and margin region with the size of the interval between circles, shown as Fig. 8 .

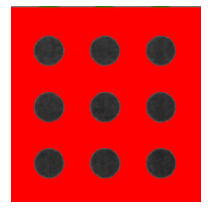

Fig. 8 Region of interest

(3) The kernel size of the Gaussian filter is reduced from 5 to 3 which makes the running time reduced. The corresponding instruction in HALCON is gauss_image (Image, ImageGauss, 3).

By taking these main measures, the running time of the program is reduced from above $200 \mathrm{~ms}$ to less than $100 \mathrm{~ms}$, which satisfies the system's requirement for realtime performance.

In addition, the accuracy of the system is verified by virtue of AAR-2 experimental platform, and the error is less than $0.6 \mathrm{~mm}$, which meets the requirement of precision.

\section{Conclusion}

The pose measurement system of AAR-2 in space is effectively modeled and simulated. After images of the cooperation target are captured in real-time, image preprocessing, image segmentation, edge extraction and ellipse fitting process are carried out to extract the centers of target circles. Then, according to the 3D coordinates of cooperation targets feature points, their corresponding 2D image coordinates, and the interior camera parameters, the position and attitude of camera relative to the target are calculated using the PnP algorithm. Furthermore, the automatic global threshold method is adopted to improve the reliability. A series of measures to improve the real time are taken, such as reducing the image, setting the interest area and reducing the kernel size of the Gaussian filter. The system is simulated in the HALCON software, and the results demonstrate its satisfactory performance in terms of computational cost and precision. 


\section{ACKNOWLEDGMENT}

The authors would like to acknowledge the support from the National Science Foundation of China under Grant No. 51775541, 51575412, 51575338 and 51575407, and the State Key Laboratory of Robotics Foundation under Grant No. Y5M3180301, Y5M3190301, and Y7M3250301, the EU Seventh Framework Programme (FP7)-ICT under Grant No. 611391, and the Research Project of State Key Lab of Digital Manufacturing Equipment \& Technology of China under Grant No. DMETKF2017003.

\section{References}

[1] Gao, M., Zhao,G., Gu,Y.: Space Science and Application Mission in China's Space Station. In: Bulletin of the Chinese Academy of Sciences. 30(6), 721-732(2015)

[2] Eslinger, G., Saenz-Otero, A.: Electromagnetic formation flight control using dynamic programming. In: Proc. of 36th Annual AAS Guid. Control Conf., 17-32(2013).

[3] Fong, T., Micire, M., Morse, T., Park, E., Provencher, C., To, V., et al.: Smart SPHERES: a Telerobotic Free-Flyer for Intravehicular Activities in Space. In: proceedings of AIAA SPACE 2013 Conference and Exposition. San Diego, CA: AIAA. 1-15(2013)

[4] Bualat, M., Barlow, J., Fong, T., Provencher, C., Smith, T., Zuniga, A.: Astrobee: Developing a Freeflying Robot for the International Space Station. In: proceedings of AIAA SPACE 2015 Conference and Exposition. Pasadena, California: AIAA. 1-10(2015)

[5] Coltin, B., Fusco, J., Moratto, Z., Alexandrov, O., Nakamura, R.: Localization from Visual Landmarks on a Free-flying Robot. In Proc. of International Conference on Intelligent Robots and Systems (IROS), IEEE /RSJ. Daejeon, Korea. 4377-4382 (2016).

[6] Liu, J. Gao, Q. Liu, Z. Li, Y.: Attitude control for astronaut assisted robot in the space station, In: International Journal of Control, Automation and Systems. 14 (4): 1082-1095 (2016).

[7] Gao, Q., Liu, J., Tian, T., Li, Y.: Free-flying dynamics and control of an astronaut assistant robot based on fuzzy sliding mode algorithm. In: Acta Astronautica. 462-474(2017)

[8] Zhang, Z.: A Flexible New Technique for Camera Calibration. In: IEEE Transactions on Pattern Analysis and Machine Intelligence. 22 (11):1330-1334(2000)

[9] Li, Z., Chen, X.: Research on Image Analysis of Bank Card Based on HALCON Software. In: Electronic Sci. \& Tech. 30(9):56-59(2017)

[10] Wang, H., Zhao, B., Sun, Z., Chen, X.: Defect Detection of Medical Bags Based on Halcon. In: Packaging Engineering, 36(7):31-35(2015)

[11] Ai, X., Xing, J. Computer Vision: Algorithms and Applications [M]. Beijing: Tsinghua University Press. 388(2012)

[12] Canny, J.: A Computational Approach to Edge Detection. In: IEEE Transactions on Pattern Analysis and Machine Intelligence. (6): 679-698(1986)

[13] ElAraby, W., Madian, A., Mahmoud, A., Farag, I., Nassef, M.: Fractional Canny Edge Detection for Biomedical Applications. In: Proceedings of IEEE International Conference on Microelectronics (ICM). 265-268(2016)

[14] Nikolic, M., Tuba, E., Tuba, M.: Edge detection in medical ultrasound images using adjusted Canny edge detection algorithm. In: Proceedings of IEEE Telecommunications Forum (TELFOR). 1-4(2016)

[15] Wang, T., Dong, W., Wang, Z.: Position and orientation measurement system based on monocular vision and fixed target. In: Infrared and Laser Engineering, 46(4):1-8(2017) 